\section{Metodología de adaptación de una guía clínica para el manejo de pacientes adultos con neumonía adquirida en la comunidad en una red de salud privada}

\author{
TOMÁS PANTOJA C..$^{1,2}$, CONSTANZA FERDINAND O. ${ }^{7, a}$, \\ FERNANDO SALDÍAS P. ${ }^{3}$, LUIS ROJAS O. ${ }^{4}$, MARÍA ELVIRA BALCELLS M. ${ }^{5}$, \\ RICARDO CASTRO L. ${ }^{6}$, RODRIGO POBLETE U. ${ }^{7}$.
}

\section{The adaptation methodology of a guideline for the management of adults with community- acquired pneumonia}

Background: Clinical practice guidelines (CPG) are widely used as tools for improving quality of health care. Guidelines developed elsewhere, can be adapted using a valid and systematic process. Aim: To describe the methodology used in the process of adaptation of a guideline for the management of adults with community-acquired pneumonia (CAP) in a private health care organization. Material and Methods: We used the ADAPTE framework involving three main phases. At the set-up phase a guideline adaptation group integrated by medical specialists from different disciplines, a methodologist and a nurse coordinator was formed. At the adaptation phase, the specific clinical questions to be addressed by the guidelines were identified. Results: Twenty five guidelines were initially retrieved. After their assessment, the number was reduced to only three. Recommendations from these guidelines were 'mapped' and focused searches were carried out where 'evidence gaps' were identified. An initial draft was written and revised by the adaptation group. At the finalization phase, the external review of the guideline was carried out and a process for the regular review and update of the adapted guideline was defined. Conclusions: We developed a guideline for the management of adults with CAP, adapted to the local context of our health care system, using guidelines developed elsewhere. This guideline creation method can be an efficient means of saving professional resources.

(Rev Med Chile 2011; 139: 1403-1413).

Key words: Pneumonia; Practice guidelines as topic; Quality of health care.

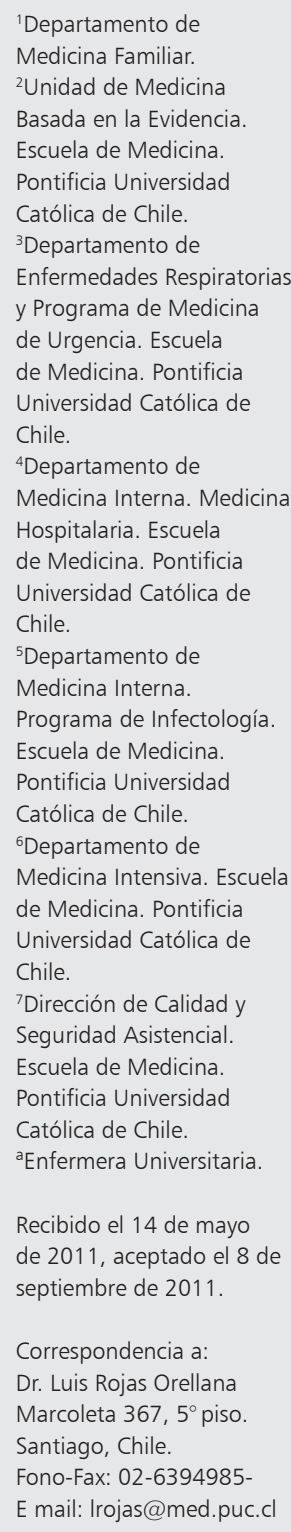

L a calidad de la atención otorgada en los sistemas de salud ha sido un tema de análisis recurrente tanto en el ámbito nacional como internacional ${ }^{1-3} \mathrm{y}$ ha sido un tema central en los esfuerzos de cambio realizados en el sector salud durante la última década en nuestro país $s^{4,5}$. Como parte de esta tendencia, la Red de Salud UC de la Facultad de Medicina de la Pontificia Universidad Católica de Chile ha enfatizado la necesidad de implementar prácticas clínicas basadas en la mejor evidencia disponible con el fin de lograr los mejores resultados esperables en nuestros pacientes. Las guías de práctica clínica (GPC) -entendidas como "conjuntos de recomendaciones elaboradas sistemáticamente para asistir las decisiones de los profesionales sanitarios y sus pacientes acerca del cuidado de salud apropiado para circunstancias clínicas específicas"- preten- 
den mejorar la eficiencia, efectividad y seguridad en la toma de decisiones, a través de la disminución de la variabilidad inapropiada en las actuaciones clínicas ${ }^{6}$. Entendiendo el complejo entorno en el que se realiza la toma de decisiones clínicas, así como la gran cantidad de información disponible para la toma de éstas, las guías pretenden apoyar a los profesionales en el proceso de cuidado de sus pacientes ${ }^{7}$.

La neumonía adquirida en la comunidad (NAC) es la principal causa de muerte por enfermedades infecciosas y la octava causa de muerte en la población adulta en nuestro país ${ }^{8}$. Se ha observado una gran variabilidad en los criterios de hospitalización por neumonía en diferentes áreas geográficas, determinando la admisión de pacientes de bajo riesgo que pudieran manejarse en el ámbito ambulatorio a un menor costo y también dificultades en la pesquisa de pacientes de alto riesgo que deberían ser manejados en unidades especializadas, lo cual afecta negativamente su evolución y pronóstico ${ }^{9,10}$. La adherencia de los médicos a las recomendaciones de las guías clínicas de neumonía se ha asociado a menor estadía en el hospital, menor costo de la atención de salud, reducción del riesgo de complicaciones y muerte en el hospital y seguimiento a 30 días ${ }^{11-13}$.

La Dirección de Calidad y Seguridad Asistencial de la Red de Salud UC se propuso a fines del año 2008 la elaboración de una guía clínica para el diagnóstico, evaluación de la gravedad, tratamiento y seguimiento de la neumonía del adulto adquirida en la comunidad. El plan inicial pretendió elaborar una GPC basado en la experiencia de organizaciones y agencias internacionales reconocidas en el área, como el Sistema Nacional de Salud Español ${ }^{14}$, National Institute for Health and Clinical Excellence (NICE) de Inglaterra ${ }^{15} \mathrm{o}$ Scottish Intercollegiate Guidelines Network (SIGN) de Escocia ${ }^{16}$. Este proceso ha sido empleado por el Ministerio de Salud chileno en la elaboración de guías clínicas para el Régimen de Garantías Explícitas en Salud (RGES). Sin embargo, dados los requerimientos humanos y financieros precisados para la elaboración de guías clínicas de alta calidad ${ }^{17}$ y la existencia de guías clínicas nacionales e internacionales en el tópico ${ }^{18-27}$, una alternativa fue la adaptación de guías elaboradas por otras organizaciones a través de un proceso válido y sistemático. El objetivo de este artículo es presentar la metodología utilizada en el proceso de adaptación de una guía de práctica clínica, con el fin de identificar aspectos que puedan ser de utilidad para implementar este tipo de proceso tanto en nuestra Red de Salud, como en otras organizaciones del sistema sanitario público o privado en nuestro país.

\section{Métodos}

En la última década se han desarrollado dos sistemas de adaptación de GPC, estableciendo el marco conceptual para este proceso y actualmente ambos grupos se han fusionado en lo que se conoce como la colaboración ADAPTE ${ }^{28,29}$. El marco metodológico definido por la colaboración ADAPTE involucra 24 etapas organizadas en tres fases principales: 1) La fase de preparación establece las áreas a ser completadas antes del proceso de adaptación propiamente tal, por ejemplo, la identificación de los recursos y competencias necesarias para la tarea; 2) La fase de adaptación asiste a los usuarios a avanzar desde la identificación del tema clínico, a la identificación de las preguntas clínicas específicas, a la búsqueda y evaluación de las guías, a la selección de la(s) guía(s) apropiadas y a la preparación del borrador de la guía adaptada; 3) La fase de finalización incluye la revisión externa de la guía adaptada, con retroalimentación de los diferentes grupos involucrados y consultas a los elaboradores de las guías utilizadas en el proceso de adaptación. Además, un proceso de revisión y actualización regular de la guía es establecido, y se edita el documento final. Este proceso es apoyado por recursos de capacitación, como un Manual y Guía Técnica que incluye herramientas de apoyo al proceso de adaptación ${ }^{30}$.

Se decidió utilizar esta aproximación al proceso de adaptación y se estableció un grupo inicial de discusión en el que participaron los directores de las áreas clínicas involucradas en el cuidado de los pacientes con esta condición, un asesor metodológico y un coordinador del grupo. Se establecieron los términos generales de referencia y se identificaron los recursos y habilidades necesarias para el establecimiento del Grupo de Adaptación. El Grupo de Adaptación es una pieza clave para el éxito del proyecto y su constitución pretende garantizar que: a) Exista representatividad de todas las áreas profesionales relacionadas; b) Toda la información científica relevante sea localizada y evaluada 
adecuadamente; c) Se identifiquen y traten los problemas prácticos derivados de la implementación de la guía; d) Exista credibilidad y aceptación de la guía entre los usuarios; e) Se reduzcan las barreras que obstaculicen la implementación de las recomendaciones de la guía. El grupo de trabajo quedó integrado por médicos especialistas en medicina interna, enfermedades respiratorias del adulto, enfermedades infecciosas, medicina de urgencia, medicina intensiva y medicina familiar (Metodólogo) bajo la coordinación técnica de una Enfermera de la Dirección de Calidad y Seguridad Asistencial de la Red de Salud UC.

\section{Etapas del proceso}

\section{Preparación}

Se estableció que el objetivo de la guía sería proporcionar recomendaciones basadas en la mejor evidencia científica disponible como referencia para el proceso asistencial de los pacientes adultos con diagnóstico de neumonía comunitaria en la Red de Salud UC. En la primera reunión del Grupo de Adaptación, se establecieron los términos generales de referencia, se presentó la metodología a utilizar (especialmente como se manejaría el proceso de discusión y consenso) y los compromisos respecto a dedicación horaria, autoría y etapas posteriores de diseminación e implementación. Se estableció la periodicidad de las reuniones y la metodología de trabajo basada en el proceso de adaptación.

Se establecieron las definiciones iniciales respecto al ámbito de la Guía Clínica:

\section{a) Condición/Patología}

La neumonía comunitaria es la infección respiratoria aguda que compromete el parénquima pulmonar ocasionado por microorganismos adquiridos fuera del ambiente hospitalario.

\section{b) Población}

Criterios de inclusión: Adultos mayores de 18 años, inmunocompetentes (incluye pacientes con terapia esteroidal $\leq 20 \mathrm{mg} /$ día de prednisona o equivalente) con neumonía adquirida en la comunidad.

Criterios de exclusión: Pacientes menores de 18 años; pacientes inmunosuprimidos secundario a infección por VIH, neoplasia hemato-oncológica activa o terapia farmacológica inmunosupresora, incluyendo corticoides a dosis mayor a $20 \mathrm{mg} /$ día de prednisona o equivalente; neumonía adquirida en el hospital o centros de pacientes crónicos u otros factores de riesgo para neumonía asociada a la atención de salud, como estadía hospitalaria reciente, uso de antibióticos recientes, hemodiálisis crónica.

\section{c) Ámbito asistencial}

c1) La guía clínica cubrirá la atención ambulatoria y hospitalaria, desde el momento en que se realiza el diagnóstico de NAC.

c2) La guía incluirá áreas que requieren coordinación entre la atención ambulatoria y hospitalaria de la Red de Salud UC.

\section{d) Proceso asistencial}

Las recomendaciones incluidas en la guía clínica han considerado los siguientes aspectos del manejo clínico: criterios diagnósticos, evaluación de la gravedad, manejo y tratamiento ambulatorio y hospitalario, criterios de alta, tratamiento kinési$\mathrm{co}$, medidas preventivas y criterios de seguimiento.

\section{Determinación de preguntas claves}

De acuerdo a las definiciones establecidas, se elaboraron las preguntas claves que la guía clínica intenta responder (Tabla 1). Para ello se definieron los siguientes cinco aspectos:

a) La Población de interés y las características de la enfermedad/condición.

b) Las Intervenciones de interés.

c) Los Profesionales a los cuáles la guía clínica está dirigida.

d) Los desenlaces (Outcomes) esperados, incluyendo aquellos relacionados con los pacientes, con la organización o con aspectos de salud pública.

e) El ámbito (Health care setting) y contexto en el cuál la guía clínica será implementada.

\section{Búsqueda de guías clínicas y otros documentos relevantes}

Se realizó una búsqueda en las bases de datos de los organismos recopiladores de guías clínicas como el US National Guidelines Clearinghouse (NGC) y la Guidelines International Network (G-I$\mathrm{N}$ ); y en organismos elaboradores de guías clínicas como el National Institute for Health and Clinical Excellence (NICE), Scottish Intercollegiate Guidelines Network (SIGN) y New Zealand Guidelines 
Tabla 1. Preguntas claves incluidas por el grupo de adaptación de acuerdo a los dominios utilizados

\begin{tabular}{|c|c|}
\hline Dominio & Detalle de pregunta clave \\
\hline $\begin{array}{l}\mathrm{P}=\text { La Población de interés y las carac- } \\
\text { terísticas de la enfermedad/condición }\end{array}$ & $\begin{array}{l}\text { Mayores de } 18 \text { años, inmunocompetentes (incluye dosis corticoides menor o igual } \\
\text { a } 20 \mathrm{mg} / \text { día de prednisona o equivalente) con neumonía comunitaria }\end{array}$ \\
\hline \multirow[t]{3}{*}{ I = Las Intervenciones de interés } & $\begin{array}{l}\text { a) Diagnóstico de neumonía } \\
\text { b) Evaluación de la gravedad ¿Cuáles serán los criterios para la clasificación de } \\
\text { gravedad de estos pacientes? }\end{array}$ \\
\hline & $\begin{array}{l}\text { Manejo en el hospital: } \\
\text { a) Criterios de ingreso a los Servicios Clínicos ¿Qué parámetros se usarán? } \\
\text { b) Criterios de tratamiento kinésico ¿Cuáles serán los criterios de solicitud de } \\
\text { tratamiento kinésico? } \\
\text { c) Criterios para el estudio microbiológico ¿Se realizará estudio microbiológico? } \\
\text { ¿A quién? ¿Cuál? } \\
\text { d) ¿Cuál es el tratamiento antibiótico empírico a utilizar? ¿A quién? ¿Cuándo? } \\
\text { e) Criterios de cambio a terapia antibiótica oral y alta } \\
\text { f) Criterios de seguimiento }\end{array}$ \\
\hline & $\begin{array}{l}\text { Manejo ambulatorio: } \\
\text { a) Tratamiento ambulatorio }\end{array}$ \\
\hline $\begin{array}{l}\text { P = Los Profesionales a los cuáles la } \\
\text { guía clínica está dirigida }\end{array}$ & Médicos clínicos de atención primaria, hospitalaria y cuidado crítico \\
\hline $\begin{array}{l}\mathrm{O}=\text { Los desenlaces (Outcomes) espe- } \\
\text { rados }\end{array}$ & $\begin{array}{l}\text { a) Disminución de la mortalidad asociado a la neumonía del adulto inmunocom- } \\
\text { petente adquirida en la comunidad } \\
\text { b) Disminución de la estadía hospitalaria y reducción de costos asociados al ma- } \\
\text { nejo de la población de riesgo elevado }\end{array}$ \\
\hline $\begin{array}{l}\text { H = El ámbito (Health care setting) y } \\
\text { contexto en el cuál la guía clínica será } \\
\text { implementada }\end{array}$ & Red de Salud UC \\
\hline
\end{tabular}

Group (NZGG) utilizando términos relevantes en texto libre (pneumonia, community-acquired pneumonia) o revisando el sitio web con la función "browse". Dicha búsqueda se complementó con una búsqueda en Medline (a través de PubMed) utilizando los términos MeSH guidelines and pneumonia; y una búsqueda manual en los registros mantenidos por algunos de los integrantes del grupo de adaptación. Mediante esta estrategia se extrajeron 25 documentos que fueron considerados relevantes para un análisis más en detalle.

\section{Tamizaje de guías clínicas obtenidas}

El tamizaje inicial de los documentos identificados en la etapa anterior, de acuerdo a criterios previamente establecidos (grupo objetivo, definición del problema, año de publicación), redujo el número de guías clínicas significativas a nueve (Tabla 2). Una revisión más exhaustiva de los documentos seleccionados que abordaban los aspectos del proceso de cuidado y respondían a las preguntas clínicas relevantes establecidas anteriormente, redujo el número de guías a ser consideradas a cinco (GP1-5). Algunos documentos fueron excluidos en esta etapa por diversas razones dentro de las que merecen ser mencionadas: a) No se incorporaba una definición clara de neumonía; b) No establecían recomendaciones respecto al diagnóstico lo que hizo difícil juzgar si se refería a la misma condición clínica de interés para el grupo; c) Sólo realizaban recomendaciones respecto al tratamiento antibiótico, lo que era insuficiente considerando el ámbito de la guía que se pretendía adaptar.

\section{Evaluación de las guías clínicas seleccionadas}

Se evaluó la calidad metodológica de las cinco guías seleccionadas utilizando un instrumento elaborado por un grupo de investigadores a nivel internacional denominado la colaboración AGREE (Appraisal of Guidelines Research \& Evaluation $)^{31}$. Este instrumento evalúa la calidad del reporte, 
Tabla 2. Listado de guías clínicas de neumonía comunitaria del adulto identificadas inicialmente

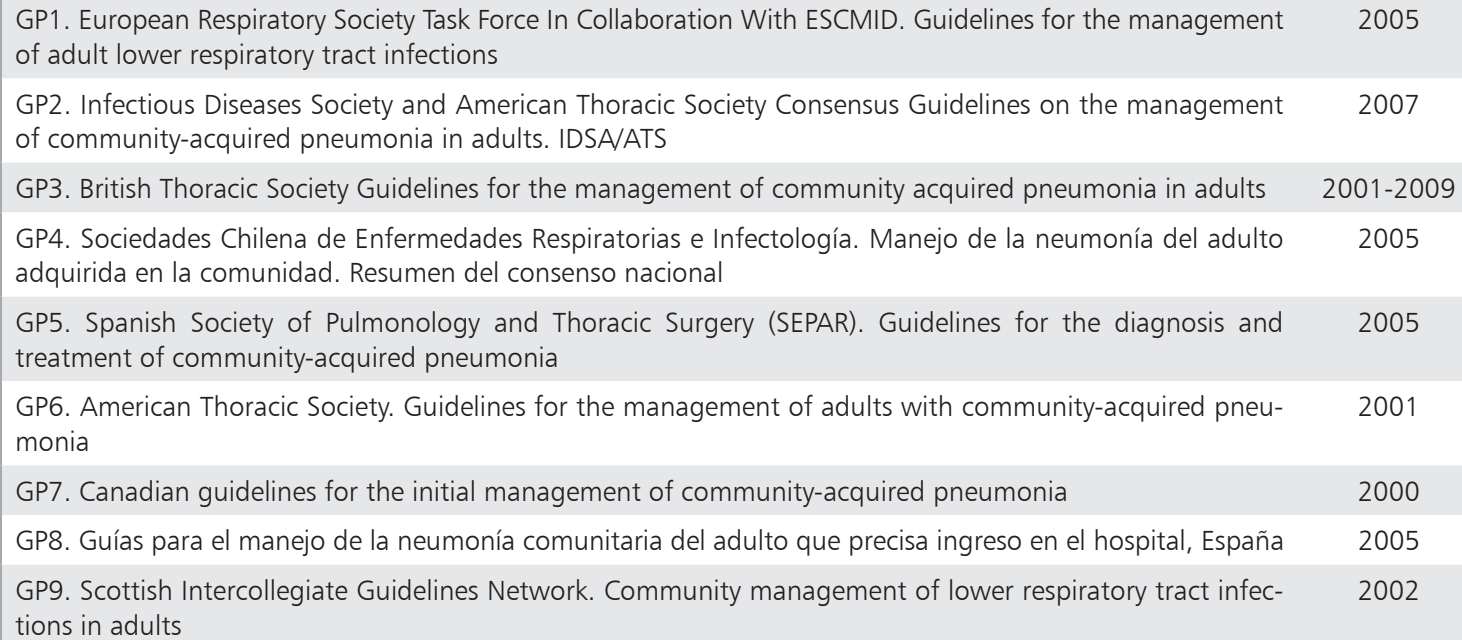

algunos aspectos de las recomendaciones y entrega una evaluación de la validez predictiva de la guía, es decir, si ésta logrará el resultado esperado. El instrumento es genérico y puede ser aplicado a guías de cualquier condición/enfermedad, incluye aspectos de diagnóstico, promoción y tratamiento. Está estructurado en 23 ítems organizados en seis dominios. Cada ítem es evaluado en una escala de Likert de 4 puntos desde 'muy de acuerdo' a 'muy en desacuerdo'. Cada dominio intenta capturar una dimensión específica de la calidad de la guía: alcance y objetivo, participación de los implicados, rigor en la elaboración, claridad y presentación, aplicabilidad e independencia editorial.

Cada integrante del Grupo de Adaptación evaluó en detalle dos guías clínicas asignadas en forma aleatoria, siguiendo las instrucciones estándar del instrumento. Dos guías fueron descartadas por presentar puntajes inferiores al $40 \%$ del máximo en la mayoría de las seis dimensiones evaluadas por el instrumento (GP4-5). Las tres guías clínicas finalmente seleccionadas para un análisis detallado de sus recomendaciones fueron:

GP1. European Respiratory Society Task Force in Collaboration with ESCMID. Guidelines for the management of adult lower respiratory tract infections, 2005.

GP2. Infectious Diseases Society of America/American Thoracic Society Consensus Guidelines on the management of community-acquired pneumonia in adults. IDSA/ATS, 2007.
GP3. British Thoracic Society Guidelines for the management of community acquired pneumonia in adults, 2001-09.

\section{Análisis de las guías clínicas}

De acuerdo a las etapas del proceso asistencial descritas previamente (Tabla 1), cada integrante del Grupo de Adaptación extrajo las recomendaciones relevantes de cada una de las guías seleccionadas en una matriz de recomendaciones diseñada ad hoc en una planilla de MSExcel. En dicha matriz fue posible "mapear" cada posible recomendación relevante para el proceso asistencial en cada una de las tres guías, lo que permitió un análisis y discusión detallada de dichas recomendaciones, así como de la evidencia detrás de ellas y su aplicabilidad en el contexto de nuestra Red de Salud.

\section{Redacción de las recomendaciones}

En base a la discusión y análisis descritos en la etapa previa se elaboró un primer listado de recomendaciones para cada una de las etapas del proceso asistencial de los pacientes adultos con NAC atendidos en la Red de Salud UC. Los desacuerdos fueron resueltos por consenso y cuando se consideró necesario se buscó y discutió nueva evidencia existente respecto a preguntas clínicas específicas. Este primer listado de recomendaciones fue examinado por el Grupo de Adaptación 
para identificar brechas específicas no discutidas o errores en la redacción o interpretación de los tópicos examinados. Luego se realizó un proceso de revisión interna utilizando la metodología Delphi $^{32}$ para evaluar el consenso existente respecto a cada una de las recomendaciones. Aquellas recomendaciones en las que no se logró consenso fueron reevaluadas, modificadas o eliminadas.

\section{Revisión externa}

El listado final de recomendaciones fue enviado para revisión externa a un grupo de revisores sugeridos por cada integrante del Grupo de Adaptación, utilizando nuevamente la metodología Delphi. El objetivo fue evaluar el grado de consenso existente entre ellos respecto a las recomendaciones específicas de la guía. Se siguió el mismo proceso detallado anteriormente para la revisión interna y se logró un listado final de recomendaciones (ver Resultados).

\section{Plan de implementación}

La implementación de las recomendaciones de esta guía clínica será responsabilidad de cada uno de los Servicios Clínicos en dónde se realiza el cuidado clínico de los pacientes objetivos de la guía con la asesoría de la Dirección de Calidad y Seguridad Asistencial de la Red de Salud UC. Para ello se identificarán las principales barreras y facilitadores a la implementación a través de informantes claves y se revisará la evidencia respecto a la efectividad de diferentes estrategias de implementación de guías clínicas en contextos similares. Se evaluará periódicamente el grado de implementación de las guías clínicas en la Red de Salud UC y las principales barreras para su óptima implementación.

\section{Plan de actualización}

Las recomendaciones de esta guía clínica debieran ser revisadas en forma continua por cada uno de los grupos clínicos participantes en su adaptación, en especial si nueva evidencia respecto al ámbito de la guía es publicada o presentada. Sin embargo, no debieran pasar más de cinco años sin que dichas recomendaciones sean revisadas en forma sistemática y detallada.

\section{Resultados}

En la Tabla 1 se describen los dominios y las preguntas clínicas examinadas en las guías clínicas de la Sociedad Europea de Enfermedades Respiratorias 2005, Sociedad Americana de Tórax y Sociedad Americana de Enfermedades Infecciosas 2007 y Sociedad Británica de Tórax 2001 y 2009.

Las recomendaciones de manejo del adulto inmunocompetente atendido por neumonía comunitaria en la Red de Salud UC se describen in extenso en la Tabla 3.

\section{Tabla 3. Recomendaciones para el manejo de pacientes adultos con diagnóstico de neumonía adquirida en la comunidad}

\section{Diagnóstico}

- Debiera sospecharse neumonía en todo paciente que se presenta con síndrome febril con síntomas de focalización respiratoria (disnea, tos, expectoración, dolor torácico)

- Debiera sospecharse neumonía en los pacientes mayores de 60 años con compromiso de conciencia, fiebre y/o comorbilidad descompensada con o sin focalización respiratoria

- En pacientes con sospecha de neumonía, se debe solicitar una radiografía de tórax para confirmar el diagnóstico

- El diagnóstico de neumonía es clínico radiológico

- En todo paciente con riesgo epidemiológico de tuberculosis pulmonar (inmigrantes de países de alta prevalencia, lesiones cavitadas, neumonía del lóbulo superior de evolución subaguda) debieran solicitarse dos baciloscopias de expectoración y cultivo Koch

- Los principales diagnósticos diferenciales del paciente con neumonía son: bronquitis aguda, gripe o influenza, EPOC/ Asma reagudizado, bronquiectasias infectadas, edema pulmonar, embolia pulmonar, cáncer pulmonar variedad bronquioloalveolar, daño pulmonar por drogas

- En todo paciente con derrame pleural paraneumónico confirmado debe realizarse punción pleural para descartar un empiema 


\section{Evaluación de la gravedad}

- En todo paciente con diagnóstico de neumonía debe realizarse una evaluación de la severidad/gravedad, dado que es clave para la apropiada planificación del manejo ambulatorio u hospitalario. Los principales criterios de gravedad que deben considerarse son: edad > 65 años, comorbilidad (respiratoria, cardiaca o neurológica crónica, diabetes, cáncer, alcoholismo, insuficiencia renal o hepática crónica, desnutrición), frecuencia cardiaca $>120$ lat/min, presión arterial $<90 / 60 \mathrm{mmHg}$, frecuencia respiratoria $>20 \mathrm{resp} / \mathrm{min}$, temperatura $>40^{\circ} \mathrm{C}$, confusión mental, compromiso radiográfico multilobar, derrame pleural, hipoxemia, nitrógeno ureico sérico (BUN) elevado, comorbilidad descompensada (ej: arritmias, isquemia miocárdica, obstrucción bronquial, hiperglicemia)

- Ninguno de los modelos predictivos disponibles permiten una categorización inequívoca de los pacientes en grupos de riesgo definitivo y deben ser considerados como una ayuda al juicio clínico

- La decisión de hospitalización es clínica. Sin embargo, dicha decisión puede ser apoyada por algunas de las herramientas objetivas de evaluación de severidad/gravedad disponibles, por ejemplo CURB-65 (Edad > 65 años, compromiso de conciencia, frecuencia respiratoria $>30 \mathrm{resp} / \mathrm{min}$, presión arterial $<90 / 60 \mathrm{mmHg}, \mathrm{BUN}>20 \mathrm{mg} / \mathrm{dL}$ )

- Se deben realizar reevaluaciones periódicas de la gravedad de todo paciente con diagnóstico de neumonía

- Todo paciente que presente algunos de los siguientes criterios debiera ser considerado para ingreso a una Unidad Pacientes Críticos: Hipotensión arterial, frecuencia respiratoria > $30 \mathrm{resp} / \mathrm{min}$, compromiso de conciencia, hipoxemia grave $\left(\mathrm{PaO}_{2} / \mathrm{FiO}_{2}<250\right)$ u oliguria

\section{Manejo ambulatorio de paciente de bajo riesgo}

- Todo paciente de bajo riesgo con diagnóstico de neumonía de manejo ambulatorio debe iniciar tratamiento antibiótico por vía oral al momento del diagnóstico y, por al menos, 7 días

- En todo paciente con diagnóstico de neumonía de manejo ambulatorio menor de 60 años y sin comorbilidades el tratamiento antibiótico de primera línea/elección debiera ser Amoxicilina en dosis altas (3 g/día)

- El tratamiento alternativo debiera ser un macrólido (e.g. Claritromicina 500 mg cada 12 horas)

- En los pacientes con diagnóstico de neumonía de manejo ambulatorio mayores de 60 años o con comorbilidades el tratamiento antibiótico de primera línea/elección debiera ser Amoxicilina/Ácido Clavulánico 825 mg cada 12 h o 500 mg cada $8 \mathrm{~h}$

- El tratamiento alternativo debiera ser una quinolona respiratoria (e.g. levofloxacina 500-750 mg/día o moxifloxacina $400 \mathrm{mg} / \mathrm{día})$

- A todo paciente con diagnóstico de neumonía de manejo ambulatorio debieran recomendársele reposo, hidratación adecuada, régimen liviano y control y manejo de la fiebre con antipiréticos SOS. Además debe informársele acerca de los signos de una evolución clínica desfavorable

- Todo paciente menor de 60 años y sin comorbilidades con diagnóstico de neumonía de manejo ambulatorio y evolución clínica favorable puede ser controlado a los 7 días de evolución

- Todo paciente mayor de 60 años o con comorbilidades con diagnóstico de neumonía de manejo ambulatorio debiera ser controlado a las 48-72 horas de iniciado el tratamiento

- Todo paciente con evolución clínica desfavorable (por ejemplo: disnea progresiva, aumento frecuencia respiratoria, decaimiento progresivo, dolor torácico, persistencia de fiebre más allá de 48 horas) debiera ser controlado precozmente

- Todo paciente con diagnóstico de neumonía de manejo ambulatorio debe recibir recomendaciones respecto a: educación antitabáquica, vacunación antiinfluenza en el período epidémico, vacunación antineumocócica en los grupos de riesgo, ingreso a programa de adicciones en pacientes alcohólicos

\section{Manejo en sala de cuidados generales de paciente no grave}

(paciente hospitalizado en una unidad de pacientes bajo régimen de cuidado habitual, baja complejidad)

- A todo paciente hospitalizado se le debieran solicitar dos hemocultivos al momento del ingreso

- A todo paciente hospitalizado con diagnóstico de neumonía y en que se pueda obtener una muestra adecuada se debiera solicitar gram y cultivo de expectoración antes de iniciar el tratamiento antibiótico

- En todo paciente con riesgo epidemiológico de tuberculosis pulmonar ( $\mathrm{VIH+}$, inmigrantes de países de alta prevalencia, lesiones cavitadas, neumonía lóbulo superior de evolución subaguda) debieran solicitarse dos baciloscopias de expectoración y cultivo de Koch

- Todo paciente con sospecha clínica de tuberculosis pulmonar debiera quedar hospitalizado en aislamiento respiratorio 


\section{Tabla 3. (continuación)}

- $\quad$ No se recomienda la solicitud de rutina de otros exámenes para estudio etiológico como antígenos en orina o serología específica

- Durante el período de brote de influenza, a todo paciente hospitalizado con neumonía se le debiera solicitar test de influenza e implementar "precaución de tipo gotitas" en espera de resultado

- Si el resultado del test de influenza es positivo el paciente debiera continuar en "precaución de tipo gotitas"

- $\quad$ Si el resultado del test de influenza es negativo, se le debiera solicitar panel viral respiratorio (influenza A y B; parainfluenza 1,2 y 3; adenovirus; virus respiratorio sincicial; metapneumovirus)

- No existen indicaciones precisas de la fisioterapia respiratoria en pacientes con neumonía, su indicación debiera ser individualizada

- En todo paciente se debe iniciar tratamiento antibiótico intravenoso tan pronto se hayan realizado los hemocultivos y obtenido la muestra de expectoración

- El tratamiento antibiótico intravenoso debiera iniciarse antes de 4-6 horas desde la admisión al hospital

- La duración total del tratamiento antibiótico debiera ser de 7 a 10 días, salvo casos especiales

- El tratamiento antibiótico inicial de elección debiera ser una cefalosporina de tercera generación (por ej. Ceftriaxona 1-2 g/día, Cefotaxima 1-2 g cada 8 h). El uso adicional de macrólidos debiera ser evaluado de acuerdo a criterios clínicos específicos (por ejemplo: fracaso de tratamiento con agentes beta-lactámicos, antecedentes epidemiológicos de agente infeccioso atípico)

- En caso de alergia a agentes betaláctamicos, el tratamiento antibiótico inicial debiera ser una quinolona respiratoria (por ej. Levofloxacina $750 \mathrm{mg} /$ día)

- Deben considerarse para cambio a tratamiento antibiótico oral aquellos pacientes que cumplan los siguientes criterios: mejoría clínica y estado de conciencia, temperatura $<37,8^{\circ} \mathrm{C}$ en dos mediciones separadas por 12 horas, frecuencia respiratoria $<24$ resp/min, frecuencia cardiaca $<100$ lat/min, tolerancia enteral mantenida por 24 horas y disminución requerimientos de oxígeno

- El tratamiento antibiótico oral de elección debiera ser una cefalosporina de 2a generación (por ej. Cefuroxima 500 mg cada 12 h), una quinolona respiratoria (por ej. Levofloxacina 500-750 mg/día) o Amoxi-clavulánico 825 mg cada 12 h o 500 mg cada 8 h

- Debiera considerarse el alta en todo paciente que ha permanecido estable clínicamente, con buena tolerancia oral después de 24 horas del cambio a tratamiento antibiótico oral, y con sus comorbilidades compensadas

- En todo paciente hospitalizado con diagnóstico de neumonía debiera considerarse al momento del alta la vacunación antiinfluenza durante el período epidémico

- En todo paciente hospitalizado con diagnóstico de neumonía debiera considerarse al momento del alta la vacunación antineumocócica

- A todo paciente fumador hospitalizado con diagnóstico de neumonía debiera recomendársele activamente la suspensión del hábito tabáquico

- A todo paciente alcohólico hospitalizado con diagnóstico de neumonía debiera recomendársele ingresar a un programa para el manejo de su adicción

\section{Manejo en uci de paciente grave}

(paciente hospitalizado en una unidad de mediana o alta complejidad)

- A todo paciente hospitalizado con diagnóstico de neumonía grave se le debieran realizar dos hemocultivos al momento del ingreso (antes de iniciar tratamiento antibiótico)

- A todo paciente hospitalizado con diagnóstico de neumonía se debiera solicitar gram y cultivo de expectoración o aspirado bronquial antes de iniciar tratamiento antibiótico

- En todo paciente con riesgo epidemiológico de tuberculosis pulmonar $(\mathrm{VIH}+$, inmigrantes de países de alta prevalencia, lesiones cavitadas, neumonía lóbulo superior de evolución subaguda) debieran solicitarse dos baciloscopias de expectoración y cultivo de Koch

- Todo paciente con sospecha de tuberculosis pulmonar debiera quedar hospitalizado en aislamiento respiratorio

- Se recomienda solicitar de rutina otros exámenes para estudio etiológico como antígenos en orina de Legionella spp y S. pneumoniae o serología específica (Mycoplasma pneumoniae, Chlamydia pneumoniae) en pacientes hospitalizados con diagnóstico de neumonía grave

- Durante el período de brote de influenza, a todo paciente hospitalizado con diagnóstico de neumonía se le debiera solicitar test de influenza 
- Si el resultado del test de influenza es positivo el paciente debiera quedar en "precaución de tipo gotitas"

- Si el resultado del test de influenza es negativo, se le debiera solicitar panel viral respiratorio

- En todo paciente hospitalizado con diagnóstico de neumonía grave se debe iniciar tratamiento antibiótico intravenoso dentro de las 2 horas siguientes al ingreso y, en lo posible, después de tomados los hemocultivos

- La duración total del tratamiento antibiótico debiera ser de 10 a 14 días, salvo en el caso de neumonía por Pseudomonas spp, Staphylococcus aureus, Legionella spp y neumonías necrotizantes (en que se puede requerir prolongar a 21 días)

- El esquema de tratamiento antibiótico inicial de elección es una cefalosporina $3^{\text {ra }}$ generación (eg: ceftriaxona, cefotaxima) asociado a una quinolona respiratoria (eg: levofloxacina, moxifloxacina)

- En caso de alergia a cefalosporinas el esquema de tratamiento antibiótico inicial debiera ser una quinolona respiratoria (eg: levofloxacina, moxifloxacina) asociado a clindamicina

- En los casos de sospecha de infección por Pseudomonas spp (colonización, daño pulmonar estructural, fibrosis quística o bronquiectasias), el esquema antibiótico inicial debiera ser cefepime o carbapenémicos asociados a una quinolona respiratoria

- De confirmarse infección por Pseudomonas aeruginosa debiera ajustarse el esquema antibiótico a Ceftazidima asociado a Ciprofloxacina o Carbapenémicos más Ciprofloxacina considerando la susceptibilidad in vitro de la cepa

- Las decisiones respecto a otras intervenciones y al traslado del paciente a una unidad de cuidado habitual debieran ser guiadas por la evolución clínica del paciente

\section{Discusión}

El presente artículo describe el proceso de adaptación de guías clínicas para el manejo de la neumonía comunitaria del adulto en una institución universitaria ${ }^{28-31}$. Como producto de este trabajo se ha elaborado una guía clínica para el manejo de los pacientes con NAC que son atendidos en la Red de Salud UC. El proceso de adaptación descrito representa el primer esfuerzo sistemático por adaptar una guía clínica para su uso en el contexto nacional. Aunque dentro del proceso de reforma del sector salud que se ha venido implementando en la última década en nuestro país, las guías clínicas parecen tener un rol central, su calidad metodológica no ha sido consistentemente evaluada. Sólo contamos con una publicación que ha evaluado la calidad de las guías clínicas de atención primaria en el contexto nacional ${ }^{33}$, elaboradas antes del año 2005, cuándo se comienzan a difundir las guías del Régimen de Garantías Explícitas en Salud.

Dado que la elaboración de guías clínicas requiere una inversión importante de recursos, una alternativa atractiva a nivel local es la adaptación de guías clínicas desarrolladas por otras organizaciones. Sin embargo, para obtener un producto de calidad es necesario utilizar un proceso sistemático y transparente como el previamente descrito.

A pesar de la utilización de este proceso, identificamos una serie de limitaciones: a) El uso eficiente de la nueva metodología requiere una importante inversión de tiempo y energía de los usuarios en el proceso de aprendizaje (curva de aprendizaje); b) Su implementación requiere la participación activa de un experto metodólogo que dirija las distintas etapas del proceso; c) Un proceso de adaptación sistemático y transparente no garantiza necesariamente el éxito en la implementación de la GPC.

Las principales limitaciones para la implementación de guías clínicas en el manejo de pacientes con neumonía comunitaria son: a) Deben considerarse las barreras y facilitadores para la implementación existentes a nivel del sistema de salud, de los profesionales sanitarios y de la población atendida; b) Las recomendaciones de las guías clínicas ayudan a sistematizar el manejo de los enfermos pero no deberían ser aplicadas en forma rígida al caso particular; el juicio clínico y la valoración del paciente no pueden ser reemplazados por una GPC; c) Una proporción significativa de las recomendaciones de las GPC aún están basadas en la opinión de expertos y en algunas áreas es necesario contar con información válida que apoye alguna de las recomendaciones; d) En los procesos de elaboración e implementación de muchas GPC no se ha considerado la opinión de los pacientes o usuarios del sistema de salud.

Consideramos que esta experiencia -aunque 
restringida a una condición particular en un contexto específico- puede representar una contribución importante de nuestra institución a una política pública actualmente en implementación (Guías Clínicas del RGES), en la que se muestra la factibilidad de adaptar guías clínicas en nuestro medio, con recursos limitados y sometidos a las presiones asistenciales de muchos prestadores privados. Las guías clínicas pueden contribuir al otorgamiento de un cuidado sanitario mejor informado por la evidencia científica disponible, pero esto sólo sucederá en la medida que ellas sean elaboradas siguiendo procesos rigurosos y documentados en forma transparente para su escrutinio por todos los actores involucrados ${ }^{7,28-30}$. El proceso de adaptación presentado en este artículo intenta contribuir a dicho esfuerzo.

\section{Referencias}

1. Bodenheimer T. The American health care system - the movement for improved quality in health care. $\mathrm{N}$ Engl $\mathrm{J}$ Med 1999; 340: 488-92.

2. Committe on Quality of Health Care in America IoM. To err is human. Washington: National Academy Press; 2000.

3. Spencer E, Walshe K. Quality and safety in healthcare in Europe: A growing challenge for policymakers. Harvard Health Pol Rev 2008; 9: 149-57.

4. Unger JP, De Paepe P, Cantuarias GS, Herrera OA. Chile's neoliberal health reform: an assessment and a critique. PLoS Med 2008; 5 (4): e79. doi:10.1371/journal. pmed.0050079.

5. Bastias G, Pantoja T, Leisewitz T, Zárate V. Health care reform in Chile. CMAJ 2008; 179: 1289-92.

6. Institute of Medicine (Field MJ \& Lohr KN eds). Guidelines for Clinical Practice. From Development to Use. Washington DC: National Academy Press; 1992.

7. Woolf SH, Grol R, Hutchinson A, Eccles MP, Grimshaw JM. Clinical guidelines: Potential benefits, limitations, and harms of clinical guidelines. BMJ 1999; 318: 527-30.

8. Ministerio de Salud de Chile. Departamento de estadísticas e información de salud (http://deis.minsal.cl/index. asp). Revisado el 25 de abril de 2011.

9. Ewig S. Community-acquired pneumonia: definition, epidemiology, and outcome. Semin Respir Infect 1999; 14: 94-102.

10. Almirall J, Bolibar I, Vidal J, Sauca G, Coll P, Niklasson B, et al. Epidemiology of community-acquired pneumonia in adults: a population-based study. Eur Respir J 2000; 15: 757-63.
11. Dean NC, Bateman KA, Donnelly SM, Silver MP, Snow GL, Hale D. Improved clinical outcomes with utilization of a community-acquired pneumonia guideline. Chest 2006; 130: 794-9.

12. Menéndez R, Reyes S, Martínez R, De la Cuadra P, Vallés JM, Vallterra J. Economic evaluation of adherence to treatment guidelines in nonintensive care pneumonia. Eur Respir J 2007; 29: 751-6.

13. Martínez R, Reyes S, Lorenzo J, Menéndez R. Impact of guidelines on outcome: the evidence. Sem Respir Crit Care Med 2009; 30: 172-8.

14. Grupo de Trabajo sobre GPC. Elaboración de Guías de Práctica Clínica en el Sistema Nacional de Salud. Manual Metodológico. Madrid (España): Plan Nacional para el Sistema Nacional de Salud del Ministerio de Salud y Consumo; 2007.

15. National Institute for Health and Clinical Excellence. The guidelines manual. London: National Institute for Health and Clinical Excellence; 2009 Jan.

16. Scottish Intercollegiate Guidelines Network. Sign 50: A guideline developer's handbook. Edinburgh: Scottish Intercollegiate Guidelines Network; 2008.

17. Burgers JS, Grol R, Klazinga NS, MAkelA M, Zaat J, for the AGREE Collaboration. Towards evidence-based clinical practice: an international survey of 18 clinical guideline programs. Int J Qual Health Care 2003; 15: 31-45.

18. Mandell LA, Marrie TJ, Grossman RF, Chow AW, Hyland $\mathrm{RH}$, and the Canadian Community-Acquired Pneumonia Working Group. Canadian Guidelines for the initial management of community-acquired pneumonia: An evidence-based update by the Canadian Infectious Diseases Society and the Canadian Thoracic Society. Clin Infect Dis 2000; 31: 383-421.

19. Niederman MS, Mandell LA, Anzueto A, Bass JB, Broughton WA, Campbell GD, et al; American Thoracic Society. Guidelines for the management of adults with community-acquired pneumonia. Diagnosis, assessment of severity, antimicrobial therapy, and prevention. Am J Respir Crit Care Med 2001; 163: 1730-54.

20. British Thoracic Society Guidelines for the management of community acquired pneumonia in adults. Thorax 2001; 56 (Suppl 4): iv1-iv64.

21. Scottish Intercollegiate Guidelines Network. Community management of lower respiratory tract infection in adults. A national clinical guideline. www.sign.ac.uk

22. Sociedad Chilena de Enfermedades Respiratorias y Sociedad Chilena de Infectología. Manejo de la neumonía del adulto adquirida en la comunidad. Resumen del consenso nacional. Rev Med Chile 2005; 133: 953-67.

23. Woodhead M, Blasi F, Ewig S, Huchon G, Leven M, 
Ortqvist A, et al. ERS Task Force in collaboration with ESCMID. Guidelines for the management of adult lower respiratory tract infections. Eur Respir J 2005; 26: 113880.

24. Spanish Society of Pulmonology and Thoracic Surgery (SEPAR). Guidelines for the diagnosis and treatment of community-acquired pneumonia. Arch Bronconeumol 2005; 41: 272-89.

25. Álvarez-Rocha L, Alós JI, Blanquer J, Álvarez-Lerma F, Garau J, Guerrero A, et al y Grupo de Estudio de la Neumonía Comunitaria Grave. Guías para el manejo de la neumonía comunitaria del adulto que precisa ingreso en el hospital. Med Intensiva 2005; 29: 21-62.

26. Mandell LA, Wunderink RG, Anzueto A, Bartlett JG, Campbell GD, Dean NC, et al; Infectious Diseases Society of America; American Thoracic Society. Infectious Diseases Society of America/American Thoracic Society consensus guidelines on the management of community-acquired pneumonia in adults. Clin Infect Dis 2007; 44 (Suppl 2): S27-72.

27. Lim WS, Baudouin SV, George RC, Hill AT, Jamieson C, Le Jeune I, et al. Pneumonia Guidelines Committee of the British Thoracic Society Standards of Care Committee. British Thoracic Society guidelines for the management of community acquired pneumonia in adults: update 2009. Thorax 2009; 64 (Suppl 3): 1-55.
28. Graham ID, Harrison MB, Brouwers M, Davies BL, Dunn S. Facilitating the use of evidence in practice: evaluating and adapting clinical practice guidelines for local use by health care organizations. J Obstet Gynecol Neonatal Nurs 2002; 31: 599-611.

29. Fervers B, Burgers JS, Haugh MC, Latreille J, MlikaCabanne N, Paquet L, et al. Adaptation of clinical guidelines: literature review and proposition for a framework and procedure. Int J Qual Health Care 2006; 18: 167-76.

30. The ADAPTE Collaboration. The ADAPTE Process: Resource Toolkit for Guideline Adaptation. Version 2.0. 2009. http://www.g-i-n.net/document-store/adapteresource-toolkit-guideline-adaptation-version-2.

31. The Appraisal of Guidelines Research and Evaluation in Europe (AGREE) Collaborative Group. Development and validation of an international appraisal instrument for assessing the quality of clinical practice guidelines: the AGREE project. Qual Saf Health Care 2003; 12: 1823.

32. Murphy MK, Black NA, Lamping DL, McKee CM, Sanderson CF, Askham J, et al. Consensus development methods, and their use in clinical guideline development. Health Technol Assess 1998; 2 (3): 1-88.

33. Pantoja T, Strain H, Valenzuela L. [Clinical practice guidelines in primary health care: A critical appraisal]. Rev Med Chile 2007; 135: 1282-90. 